$\stackrel{N / N}{=}$

Global burnals Inc.

if

\title{
A Comparative Study of Outcomes of Treatments for Radiculopathies Arising out of Single Level Disc Prolapse of Lumbar Spine Treated with Epidural Steroid Injections Versus Conservative Management
}

By Amit Garud, Pradeep Kulkarni, Nishant Gaonkar \& Vishal Sharma Abstract- Introduction: Low back pain is one of the major causes of disability in people in 4th and 5th decade of life. Lumbar spinal stenosis can induce radiating pain in the lower limbs. Pain can be caused due to mechanical compression or nerve root irritation due to prolapse which causes radiculitis. Hence, in early disc degenerations, apart from conservative management, Epidural steroid injections have been used as an add-on in treating radiating pain as they have a potent anti-inflammatory property.

Materials and method: A total of one hundred patients with low back pain with unilateral or bilateral radiculopathy with documented with lumbar degenerative disc disease without any evident instability or neurodeficit were considered for our study. Patients were randomized and selected for further intervention for conservative management or for epidural steroid injections under fluoroscopic guidance. Results were evaluated by comparing the Visual analogue scale scores and Modified Roland Sciatica Scores at the end of six months.

Keywords: epidural steroid injection, single level disc prolapse, lumbar degenerative disc disease.

GJMR-H Classification: NLMC Code: WE 725

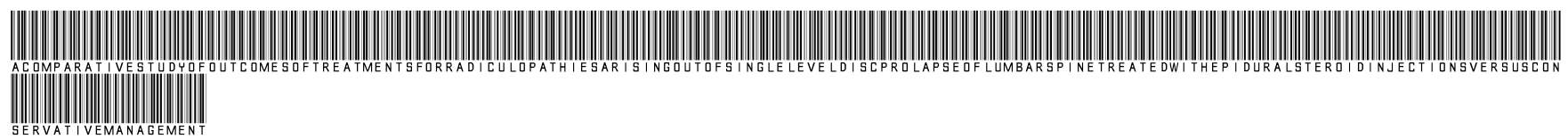

Strictly as per the compliance and regulations of:

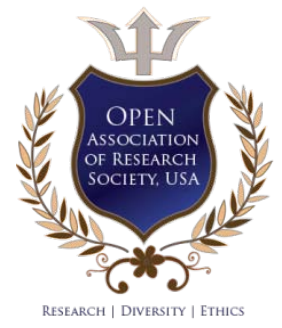

(C) 2019. Amit Garud, Pradeep Kulkarni, Nishant Gaonkar \& Vishal Sharma. This is a research/review paper, distributed under the terms of the Creative Commons Attribution-Noncommercial 3.0 Unported License http://creativecommons.org/licenses/by$\mathrm{nc} / 3.0 /$ ), permitting all non-commercial use, distribution, and reproduction in any medium, provided the original work is properly cited. 


\title{
A Comparative Study of Outcomes of Treatments for Radiculopathies Arising out of Single Level Disc Prolapse of Lumbar Spine Treated with Epidural Steroid Injections Versus Conservative Management
}

\author{
Amit Garud ${ }^{\alpha}$, Pradeep Kulkarni $^{\sigma}$, Nishant Gaonkar ${ }^{\circ} \&$ Vishal Sharma ${ }^{\omega}$
}

\begin{abstract}
Introduction: Low back pain is one of the major causes of disability in people in 4th and 5th decade of life. Lumbar spinal stenosis can induce radiating pain in the lower limbs. Pain can be caused due to mechanical compression or nerve root irritation due to prolapse which causes radiculitis. Hence, in early disc degenerations, apart from conservative management, Epidural steroid injections have been used as an add-on in treating radiating pain as they have a potent antiinflammatory property.
\end{abstract}

Materials and method: A total of one hundred patients with low back pain with unilateral or bilateral radiculopathy with documented with lumbar degenerative disc disease without any evident instability or neurodeficit were considered for our study.Patients were randomized and selected for further intervention for conservative management or for epidural steroid injections under fluoroscopic guidance. Results were evaluated by comparing the Visual analogue scale scores and Modified Roland Sciatica Scores at the end of six months.

Observations: In this study, it was observed that there was a significant decrease in pain and radiculopathy scores after immediately steroid injections which retained upto the entire study period of 6 months. In case of conservative management, the reduction in scores was more gradual.

Conclusion: Epidural steroid injections are an effective way for treating radiculopathy due to single level disc prolapse in lumbar spine as it reduces pain and radiculopathy early which encourages patients for active physiotherapy.

Keywords: epidural steroid injection, single level disc prolapse, lumbar degenerative disc disease.

\section{INTRODUCTION}

L ow back pain and spine related impairments are one of the major concerns in $4^{\text {th }}$ and $5^{\text {th }}$ decade of life and has ranked as one of the most frequent cause leading to limitation of activity ${ }^{1}$. Lumbar spinal stenosis can induce radiating pain in the lower limbs through herniated intervertebral disc, thickening

Author a w: Senior Resident, Department of Orthopedics, KIMSDU, Karad. e-mail: amitgarud12@gmail.com

Author o: Professor and Head, Department of Orthopedics, KIMSDU, Karad.

Author p: Associate Professor, Department of Orthopedics, KIMSDU, Karad. ofligamentumflavum, zygapophyseal joint and surrounding soft tissues ${ }^{2}$. Conservative management has been used widely for disc degenerative conditions. However, in many cases, structural abnormalities are not the only cause of pain, rather nerve root irritation due to disc prolapse and inflammation around nerve root causes radiculitis instead of direct mechanical compression ${ }^{3,4}$.

Steroids are well known for its efficient antiinflammatory properties ${ }^{5}$. Therefore, in the management of radicular pain produced by spinal stenosis, injected steroid is expected to contribute to pain reduction by interrupting the synthesis of prostaglandins, which in turn blocks the conduction of nociceptive-c fibers and controlling edema around the nerve root ${ }^{5}$. Hence, role of epidural steroids is that it can provide pain relief during the recovery of disc or nerve root injuries and allow patients to increase their level of physical activity.

Complications that can occur in patients receiving epidural steroid injections are injection site abscess, epidural hematoma and puncture of the dura. However, the most adverse immediate reaction during an epidural injection that can occur is vasovagal reaction. Headache without any evident dural puncture still can be seen in about $2 \%$ of patients and injection of air in the epidural space, increased intrathecal pressure due to fluid, possibly an undetected dural puncture are said to be the important causes. Accidental intravascular injections can occur because absence of blood after negative aspiration is a method that cannot be completely relied upon. Other minor complications can be non-positional headaches, facial flushing,lowgrade fever, and transient increased back pain or radiculopathy. ${ }^{6}$

Epidural steroid injections are contraindicated if there is an evident systemic or local infection at the injection site, bleeding diathesis, uncontrolled diabetes mellitus, and congestive heart failure. If there are any unexpected anatomical anomalies, such as a midline epidural septum or multiple separate epidural 
compartments, the desired flow of epidural injectants to the presumed pain generator can be restricted. ${ }^{6}$

\section{il. Materials and Methods}

The study was conducted at a tertiary care hospital from November 2016 to November 2018.

\section{a) Inclusion criteria}

1. Patients with single level disc prolapse of lumbar spine having unilateralor bilateral radiculopathy.

2. Patients with clinical correlation of radiological finding.

3. Adults of either sex.

b) Exclusion criteria

1. Patients with clinical and radiological evidence of instability like spondylolisthesis or spondylolysis.

2. Patients with traumatic spine injury.

3. Patients with previous history of lumbar surgery or epidural injections.

4. Patients with neurological deficit.

5. Patients with relevant co-morbidities.

6. Patients with known allergy to the injecting materials.

A total of hundred patients who fulfilled the above mentioned criteria were included in the study. Patients to participate in this study were documented.

There were two groups created

- Epidural steroid injection group

- Conservative management group

Patients were randomized and allotted one group for the study. So, there were 50 patients in each group. Patients were asked to record their pain and radiculopathy status using Visual analogue scale and Modified Roland Sciatica Questionnaire. Each patient was counseled about the management, expected prognosis and probable complications.

Epidural injections were given under fluoroscopic guidance by initially injecting non-ionic contrast material using the technique of safe triangle targeting the exiting root. Once the correct placement is confirmed, a cocktail of triamcinolone acetate $40 \mathrm{mg} / 1 \mathrm{cc}$ with $0.25 \mathrm{mg}$ of Bupivacaine is injected at the target site. After one hour, their pain and radiculopathy status was recorded using Visual Analogue scale (VAS). Immediate post injection Modified Roland sciatica (MRS) scores cannot be recorded as it is a lifestyle scoring system. Patients were discharged usually on the same day with pregabalin $75 \mathrm{mg} \mathrm{HS}$. Patients treated conservatively were prescribed routine non-steroidal anti-inflammatory drugs with pregabalin in increasing dosage of a maximum of $450 \mathrm{mg} /$ day.

Patients in both groups were advised active physiotherapy with lumbar exercises to strengthen lumbar paraspinal muscles, postural improvements and to improve disc biomechanics. Patients were explained about the necessary precautions like avoiding forward bending, heavy weight lifting, excessive two wheeler travelling, etc., which is likely to worsen the disease. Patients were asked to report back after one, three and six months routinely or as and when patient finds it a need to visit their doctor. Patients were again asked to report their pain using VAS scale and their radiculopathy status using MRS Questionnaire. Patients who had developed any neurological deficit in the course of study or those who came with evident instability on follow up were dropped out of study and was reconsidered for appropriate further management.

\section{ili. Observations and Results}

A prospective, comparative clinical study was conducted with 100 patients. There were equal number of patients in each group consisting of 50 patients. In epidural injection group, mean VAS score pre-injection in was 5.64(+/- 0.86) and mean MRS score pre-injection was $51.84(+/-12.91)$ while in conservative group, at the beginning of treatment, mean VAS score was $5.80(+/-0.86)$ and mean MRS score was $49.40(+/-12.20)$. None of the distributions were significant at the pre-treatment stage. (Table 1 and 2)

Table 1: VAS scores comparison within each group and between the two groups

\begin{tabular}{|c|c|c|c|c|c|}
\hline \multirow{2}{*}{ VAS Score } & \multicolumn{2}{|c|}{ Epidural Group } & \multicolumn{2}{c|}{ Conservative group } & $\begin{array}{c}\text { Comparison between } \\
\text { two groups }\end{array}$ \\
\cline { 2 - 5 } & Mean & SD & Mean & SD & P value \\
\hline Pre-treatment & 5.64 & 0.860 & 5.80 & 0.866 & $>0.05$ \\
\hline Immediate post treatment & 2.04 & 0.889 & - & - & - \\
\hline 1 month & 2.88 & 0.881 & 4.68 & 0.900 & $<0.05$ \\
\hline 3 month & 3.12 & 1.236 & 4.24 & 1.393 & $<0.05$ \\
\hline 6 month & 2.76 & 1.165 & 3.94 & 1.361 & $<0.05$ \\
\hline
\end{tabular}


Table 2: MRS scores comparison within each group and between the two groups

\begin{tabular}{|c|c|c|c|c|c|}
\hline \multirow{2}{*}{ MRS Score } & \multicolumn{2}{|c|}{ Epidural Group } & \multicolumn{2}{c|}{ Conservative group } & $\begin{array}{c}\text { Comparison between } \\
\text { two groups }\end{array}$ \\
\cline { 2 - 5 } & Mean & SD & Mean & SD & P value \\
\hline Pre-treatment & 51.84 & 12.912 & 49.40 & 12.207 & $>0.05$ \\
\hline 1 month & 32.20 & 13.454 & 43.40 & 13.156 & $<0.05$ \\
\hline 3 months & 34.20 & 13.191 & 42.80 & 13.799 & $<0.05$ \\
\hline 6 months & 34.68 & 14.673 & 39.72 & 12.628 & $<0.05$ \\
\hline
\end{tabular}

In our study, we observed that after immediate post-injection, mean VAS score in epidural injection group was 2.04+/-0.89 These scores were reduced significantly as compared to pre-injections $(p<0.05)$.

At $1^{\text {st }}$ month follow up, mean VAS score was $2.88+/-0.88$ in epidural injection group and $2.68+/-0.90$ in conservative group. When compared to immediate post injection, it was observed that mean VAS score had increased significantly $(p<0.05)$ in epidural injection group. Mean MRS score at 1st month follow up was 32.20+/-13.45 epidural injection group and $33.40+/-13.16$ in conservative group. When compared to pre-injection, it was observed that there was a significant reduction in mean MRS score $(p<0.05)$ in epidural injection group. However, in conservative group, mean VAS score at the end of $1^{\text {st }}$ month was
$4.68+/-0.900$ which was decreased significantly $(p<0.05)$ as compared to pre-treatment score. Similarly, Mean MRS score at the end of $1^{\text {st }}$ month was $43.40+/-13.15$ which was decreased significantly $(p<0.05)$ as compared to pre-treatment score. Reduction in VAS scores and MRS scores were better in Epidural group as compared to the conservative management.

On three and six months follow up, both pain and radiculopathy scores remained similar in both the groups without being statistically significant as compared to their previous scores within the group. However, the difference in the two groups was still persistent and was significant statistically. (Graphs 1 and 2)

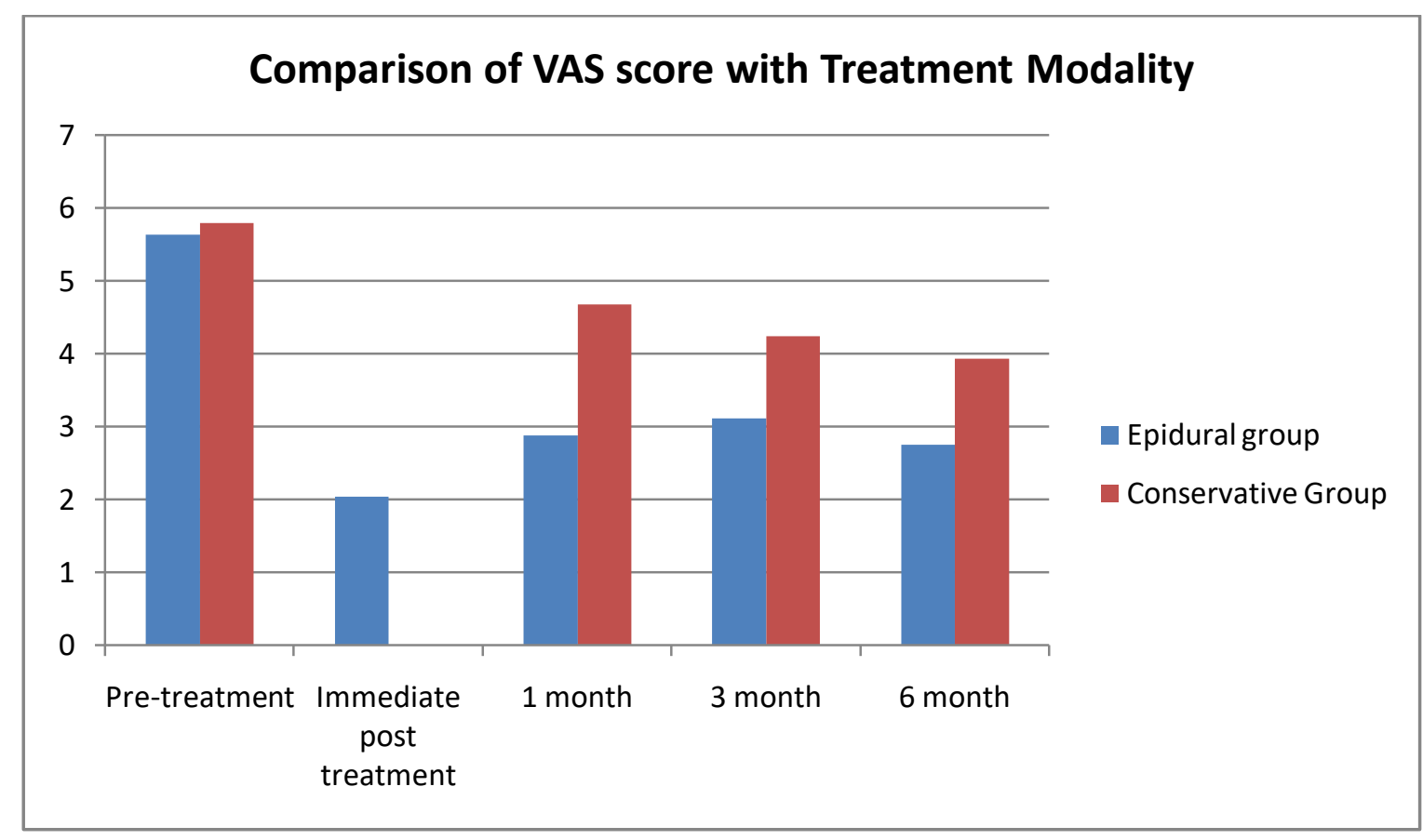

Graph 1: Comparison of VAS score with Treatment Modality 


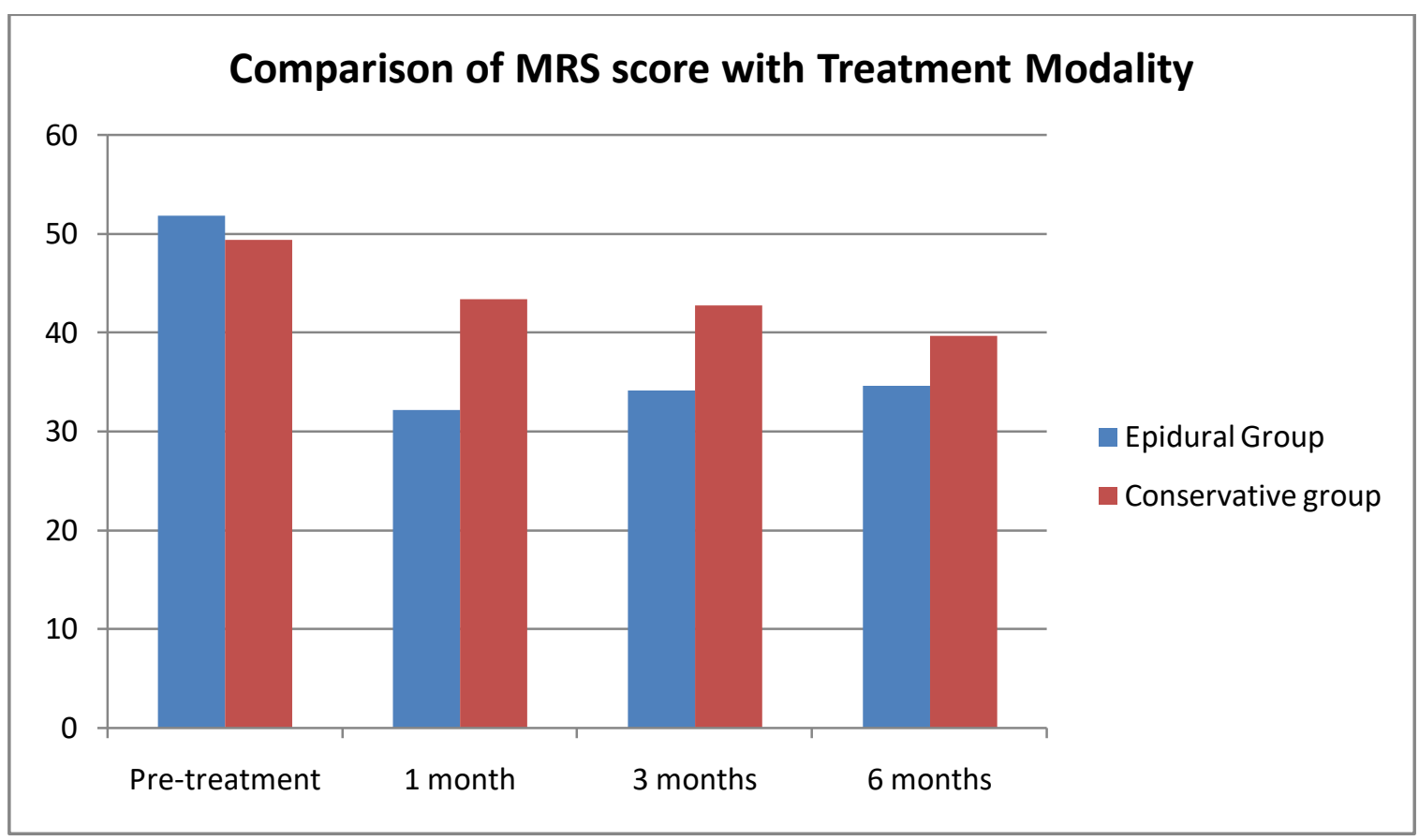

Graph 2: Comparison of MRS score with Treatment Modality

On comparing the end result of the two modalities (Graph 3), we observed that there was still a significant reduction the pain scores and radiculopathy scores in epidural injection group which was better as compared to conservative management. $(p<0.05)$.

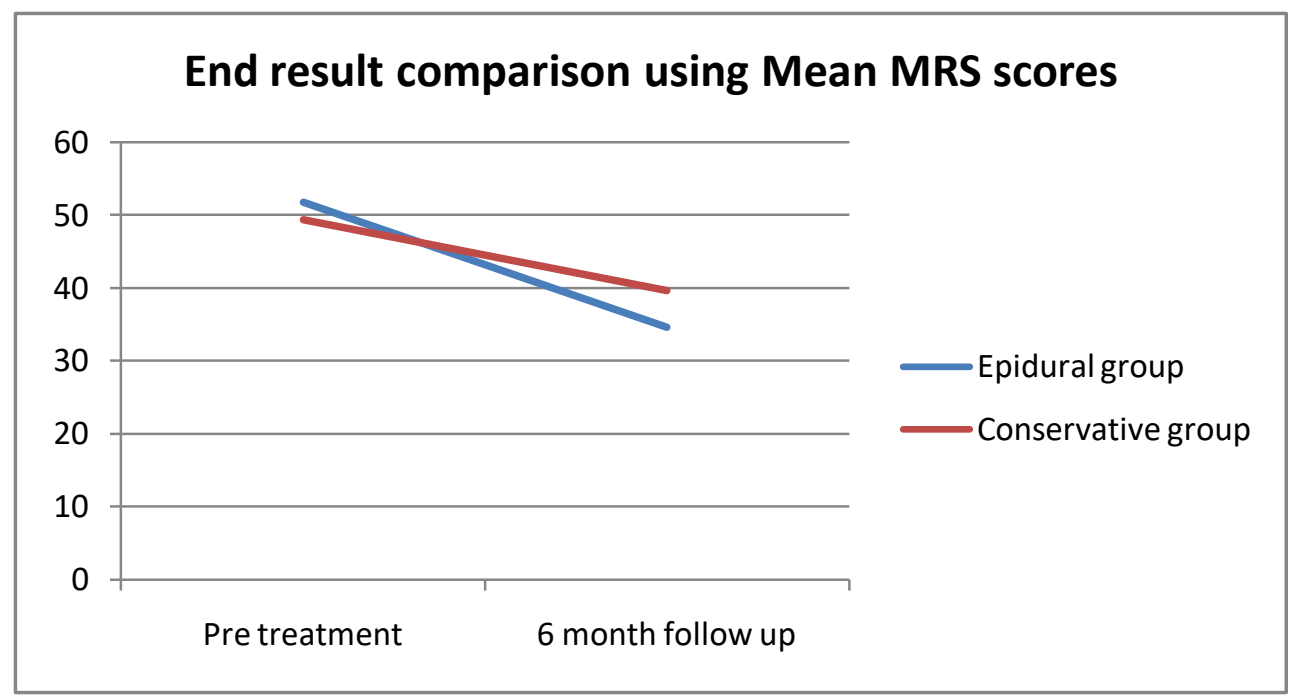

Graph 3: End Result comparison

In our study, none of the patients had any major complication. Two patients in epidural injection group had post injection headache which resolved spontaneously without the need of any active intervention.

\section{Discussion}

When we observed the trend of scores and their improvement patterns in both the groups, we can see that there was a major improvement in scores immediate post-injection as compared to pre-injection which carried away with follow up with minor variations in epidural injection group. In case of conservative management, we also observed that there was a significant improvement at the end of 6 months, but this improvement was gradual as compared to the injection group

However, at the end of six months, when we compared the difference in the two groups, we can clearly see that there was a significant improvement in scores at the end of six months when compared with pre-treatment and both the treatment modalities were 
effective but the scores at the end of six months were found to be significantly better in epidural injection group as compared to conservative management.

In our study, two patients had post injection headache in epidural group. There are various possible causes like an un-noticed dural puncture, which is less likely as it resolved spontaneously. Other probable cause could be injection of air into the epidural space.

In our study, a significant number of patients retained their improved scores throughout the study period. Various probable mechanisms which may have a role in improvement could be the steroids and bupivacaine solution, both have anti-nociceptive and nerve membrane stabilizing properties; the washout effect of the solution, which decreases the regional levels of inflammatory mediators; hydrodilation effect of the solution breaking the adhesions around the nerve root; steroids having a potent anti-inflammatory property; rehabilitation protocol which improves the biomechanics on the discs ${ }^{7,8}$.

In our study, we have chosen single dose injections as our protocol because repeated injections of steroids and local anesthetics may mask the symptoms of disease progression or development of instability.

In case of epidural steroid injections, as the cocktail of steroid and local anesthetic act directly over the site of inflammation, patients are relieved almost instantly and are more enthusiastic to follow the physiotherapy protocol which is not the case in conservative management which takes a while hence difficult and reluctant to comply with the physiotherapy.

\section{Conclusion}

Epidural steroid injections can be considered to be effective in treating radiculopathies arising out of early stages of prolapse intervertebral discs of lumbar spine. The short term results of epidural steroid injection were found to be superior as compared to conservative management. However, a good post injection protocol is necessary for good results and a close follow up is must to watch for disease progression and to avoid deterioration.

Conflict of interest: None

\section{Bibliography}

1. Guild D G (2012) Mechanical therapy for low back pain. Prime Care 39(3): 511-516.

2. Alexander C E, Varacallo M. Lumbosacral Radiculopathy. [Updated 2019 Mar 23]. In: StatPearls [Internet]. Treasure Island $(\mathrm{FL})$ : Stat Pearls Publishing; 2019.

3. Rydevik B, Brown M D, Lundborg G. Pathoanatomy and pathophysiology of nerve root compression. Spine 1984; 9: 7-15.
4. Olmarker K, Redevik B, Holm S. Edema formation in spinal nerve roots induced by experimental, graded compression. An experimental study on the pig caudaequina with special reference to differences in effects between rapid and slow onset of compression. Spine 1989; 14: 569-573.

5. Safiya Shaikh, Himanshu Verma, Nirmal Yadav, Mirinda Jauhari, and Jyothi Bullangowda, "Applications of Steroid in Clinical Practice: A Review," ISRN Anesthesiology, vol. 2012, Article ID 985495, 11 pages, 2012.

6. Azar, F., Beaty, J. and Canale, S. (2017). Campbell's operative orthopaedics. $13^{\text {th }}$ ed. Philadelphia: Elsevier Mosby, pp.1652-1656.

7. Benoist $M$, Boulu $P$, Hayem G. Epidural steroid injections in the management of low-back pain with radiculopathy: an update of their efficacy and safety. Eur Spine J. 2012; 21(2): 204-213.

8. Weinstein S M, Herring S A, Derby R. Contemporary concepts in spine care: Epidural steroid injections. Spine1995; 20: 1842-6. 